\title{
Photoacoustic microscopy of human teeth
}

Bin Rao, Xin Cai, Christopher Favazza, Junjie Yao, Li $\mathrm{Li}$, et al.

Bin Rao, Xin Cai, Christopher Favazza, Junjie Yao, Li Li, Steven Duong, Lih-Huei Liaw, Jennifer Holtzman, Petra Wilder-Smith, Lihong V. Wang, "Photoacoustic microscopy of human teeth," Proc. SPIE 7884, Lasers in Dentistry XVII, 78840U (17 February 2011); doi: 10.1117/12.874070

SPIE. Event: SPIE BiOS, 2011, San Francisco, California, United States 


\title{
Photoacoustic microscopy of human teeth
}

\author{
Bin Rao ${ }^{1}$, Xin Cai ${ }^{1}$, Favazza, Christopher ${ }^{1}$, Junjie Yao ${ }^{1}, \mathrm{Li} \mathrm{Li}^{1}$, \\ Steven Duong ${ }^{2}$, Lih-huei Liaw ${ }^{2}$, Jennifer Holtzman ${ }^{2}$, Petra Wilder-Smith ${ }^{2}$, and Lihong V. Wang ${ }^{1}$ \\ ${ }^{1}$ Department of Biomedical Engineering, Optical Imaging Lab, Washington University in St. Louis, \\ Campus Box 1097, One Brookings Drive, St. Louis, Missouri 63130-4899, USA \\ ${ }^{2}$ Beckman Laser Institute, University of California, Irvine, Irvine, CA 92612-3070, USA
}

\begin{abstract}
Photoacoustic microscopy (PAM) utilizes short laser pulses to deposit energy into light absorbers and sensitively detects the ultrasonic waves the absorbers generate in response. PAM directly renders a three-dimensional spatial distribution of sub-surface optical absorbers. Unlike other optical imaging technologies, PAM features label-free optical absorption contrast and excellent imaging depths. Standard dental imaging instruments are limited to X-ray and CCD cameras. Subsurface optical dental imaging is difficult due to the highly-scattering enamel and dentin tissue. Thus, very few imaging methods can detect dental decay or diagnose dental pulp, which is the innermost part of the tooth, containing the nerves, blood vessels, and other cells. Here, we conducted a feasibility study on imaging dental decay and dental pulp with PAM. Our results showed that PAM is sensitive to the color change associated with dental decay. Although the relative PA signal distribution may be affected by surface contours and subsurface reflections from deeper dental tissue, monitoring changes in the PA signals (at the same site) over time is necessary to identify the progress of dental decay. Our results also showed that deep-imaging, near-infrared (NIR) PAM can sensitively image blood in the dental pulp of an in vitro tooth. In conclusion, PAM is a promising tool for imaging both dental decay and dental pulp.
\end{abstract}

\section{Introduction}

Photoacoustic microscopy (PAM) utilizes short laser pulses to deposit energy into light absorbers and sensitively detects the ultrasonic waves the absorbers generate in response. PAM directly renders a three-dimensional spatial distribution of sub-surface optical absorbers. 
Unlike other optical imaging technologies, PAM features label-free optical absorption contrast and super imaging depths [1-2]. Standard dental imaging instruments are limited to X-ray and digital imaging cameras. Subsurface optical dental imaging is difficult due to the highlyscattering enamel and dentin tissue. Thus, very few imaging methods can detect dental decay or diagnose dental pulp, which is the innermost part of the tooth, containing the nerves, blood vessels, and other cells. Here, we conducted a feasibility study on imaging dental decay and dental pulp with PAM.

\section{Materials and methods}

Three PAM imaging systems were used to image in vitro dental tissues. Extracted human teeth were imaged, and their health status (ie healthy vs decayed) was determined by conventional microscopy. The first imaging system was the optical-resolution PAM imaging system [3] shown schematically in Fig. 1. The laser pulse width was $6.5 \mathrm{~ns}$. $570 \mathrm{~nm}$ laser of $40 \mathrm{~nJ}$ pulse energy was used for the experiments. The calibrated lateral resolution was $3.5 \mu \mathrm{m}$. A $75 \mathrm{MHz}$ acoustic transducer with acoustic NA of 0.45 was used to detect PA signals. The axial resolution was 20 $\mu \mathrm{m}$, and the A-scan imaging speed of the system was about $600 \mathrm{~A}$-lines per second.

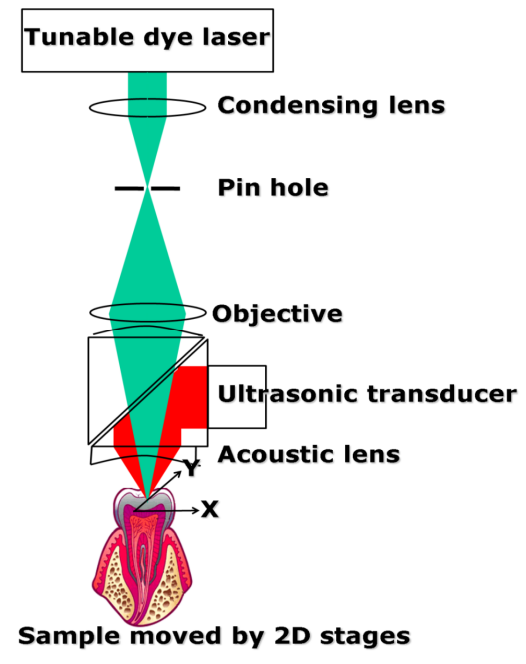

Fig.1. An optical-resolution photoacoustic microscopy setup

The second imaging system was the acoustic-resolution photoacoustic imaging system (AR-PAM) shown in Fig. 2 [4]. The laser pulse width was $6.5 \mathrm{~ns}$, the wavelength was $570 \mathrm{~nm}$, and the laser pulse energy used was $0.8 \mathrm{~mJ}$. A $50 \mathrm{MHz}$ ultrasonic transducer with acoustic NA of 0.44 was used for the detection of PA signals. The imaging system had a lateral resolution of 
$45 \mu \mathrm{m}$ and an axial resolution of $15 \mu \mathrm{m}$. The A-scan imaging speed of the system was about 600 A-lines per second.

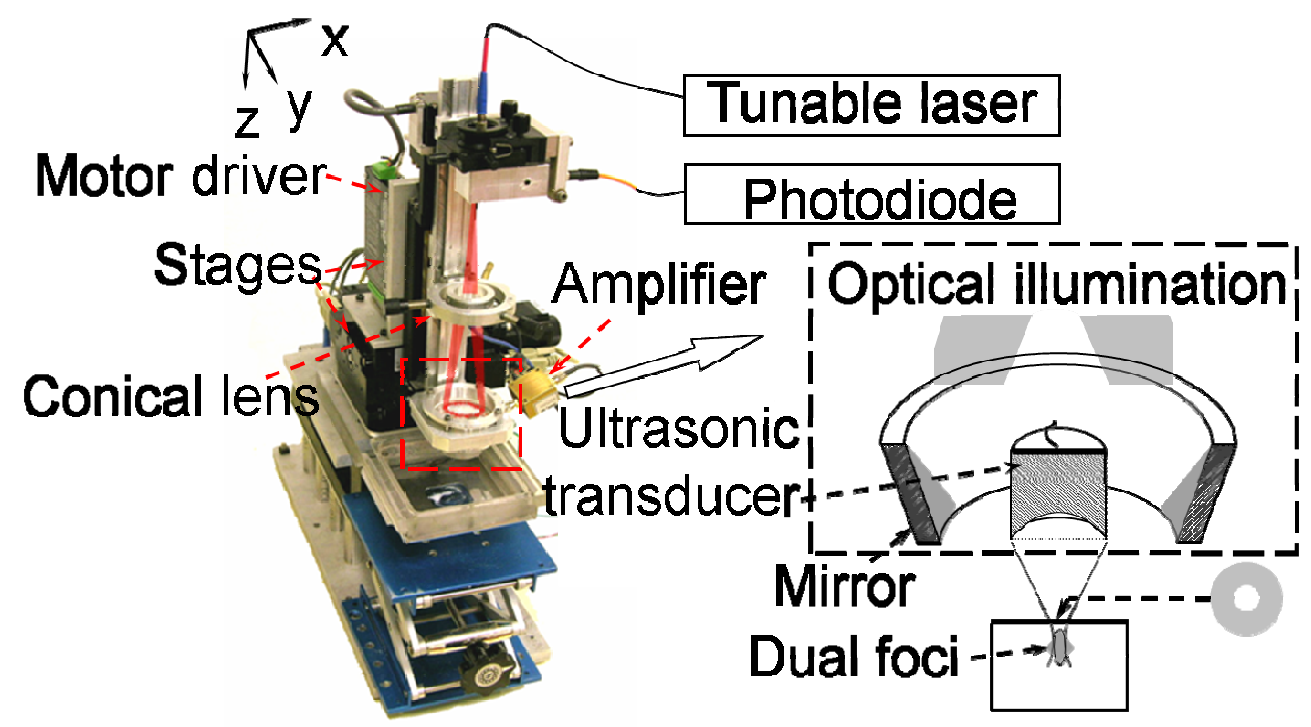

Fig. 2. An acoustic-resolution photoacoustic microscopy (AR-PAM) setup

The third imaging system was the deep imaging, NIR, acoustic-resolution photoacoustic system shown schematically in Fig. 3 [5]. The laser pulse width was 15 ns, the laser pulse energy was $60 \mathrm{~mJ}$, and the laser wavelength was $804 \mathrm{~nm}$. A $5 \mathrm{MHz}$ ultrasonic transducer with acoustic NA of 0.375 was used for the detection of PA signals. The imaging system had a lateral resolution of $500 \mu \mathrm{m}$ and an axial resolution of $300 \mu \mathrm{m}$. The A-scan imaging speed of the system, which was limited by the available laser system, was about $10 \mathrm{~A}$-lines per second.

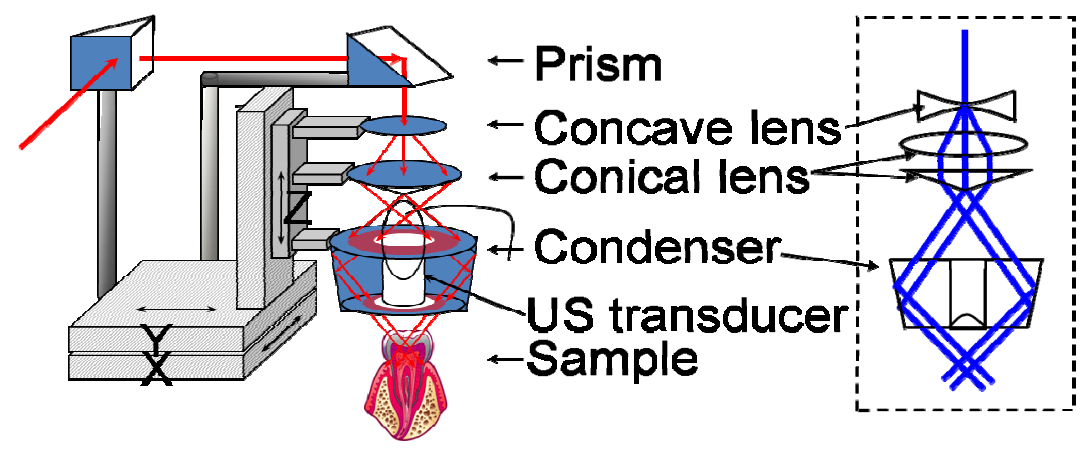

\section{Results}

Fig. 3. A deep, NIR, acoustic-resolution photoacoustic microscopy setup

In order to determine whether PAM is sensitive to color change associated with dental decay, dental tissue slices ( $2 \mathrm{~mm}$ thick) with dental decay lesions were prepared and imaged with the 
AR-PAM setup shown in Fig. 2. The color change associated with dental decay at lesion site as shown in a conventional light microscopy image (red arrow in Fig. 4 left) was clearly identified by dark-field AR-PAM in a maximum amplitude projection (MAP) image (red arrow in Fig. 4 right) with high contrast. Each two-dimensional MAP image (in an X-Y plane) was projected (along $Z$ direction) from a three-dimensional PAM image. At $532 \mathrm{~nm}$ wavelength, most of the PA signals come from absorption sites close to the surface although PAM is a threedimensional imaging modality.
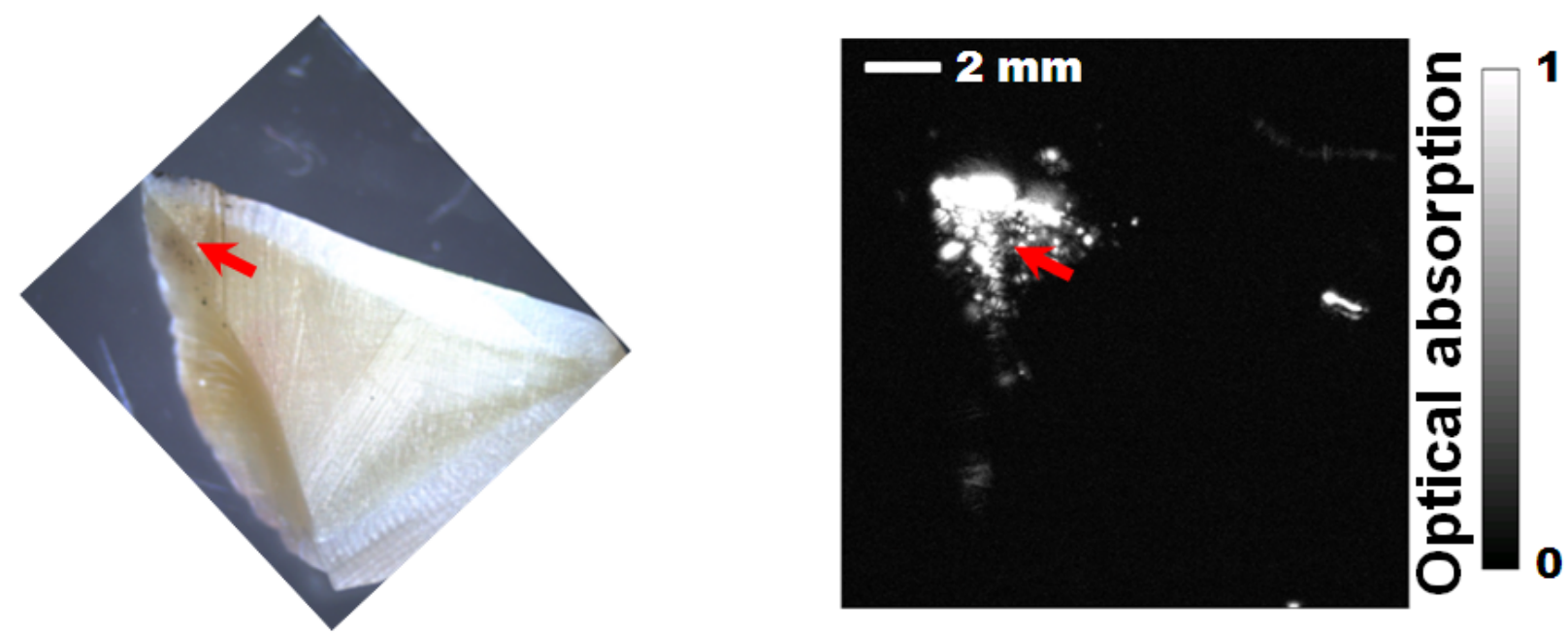

Fig. 4. The color change associated with dental decay at lesion site as shown in a conventional light microscopy image (red arrow in left) was clearly identified by dark-field AR-PAM in a maximum amplitude projection (MAP) image (red arrow in right) with high contrast.

In order to investigate dental decay, a healthy tooth, shown in the photograph (Fig. 5A), and a decayed tooth, shown in the photograph (Fig. 5B) were both imaged by the dark-field ARPAM. The dark-field AR-PAM MAP images of the healthy tooth (Fig. 5C) and the decayed tooth (Fig. 5D) showed significant differences in optical absorption contrast. The laser pulse energy was scattered by the uneven surface, and the ultrasonic wave coupling into the ultrasonic transducer was affected by both the contours of the dental surface and the complex subsurface structure. Thus, relative PA signal differences between two sites do not necessarily give decay information. Although much stronger PA signals were detected from the decayed sample, monitoring changes in the PA signals (at the same site) over time is necessary to identify the progress of dental decay. It was also found that similar results can be acquired by an opticalresolution PAM (Fig. 1). At $532 \mathrm{~nm}$ wavelength, most of the PA signals come from absorption 
sites close to the surface. But PA signals may be excited deep inside the dental tissue with NIR lasers as we demonstrated later.

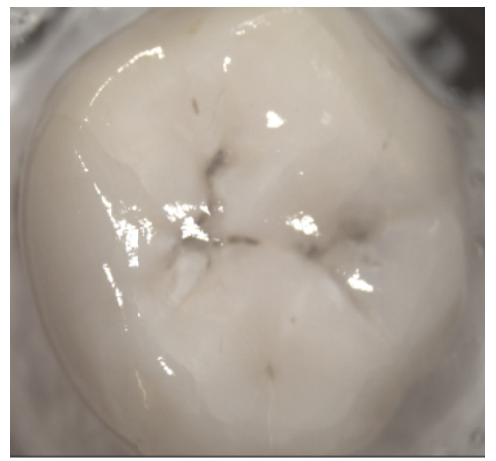

(A)

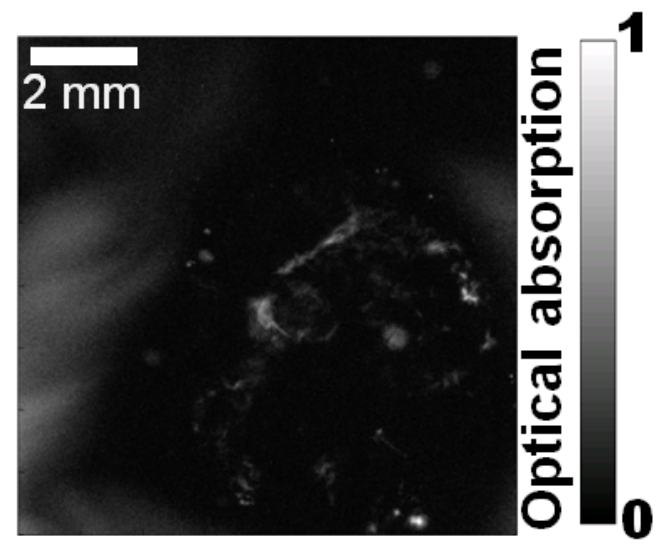

(C)

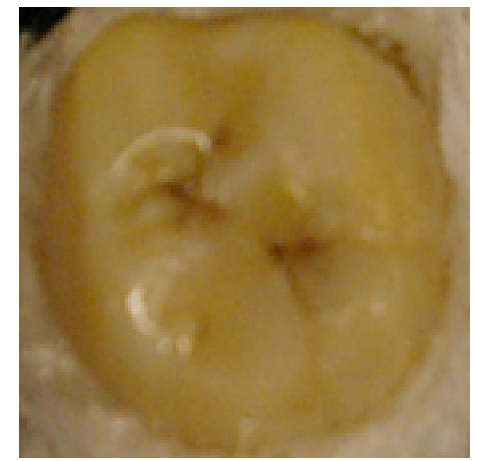

(B)

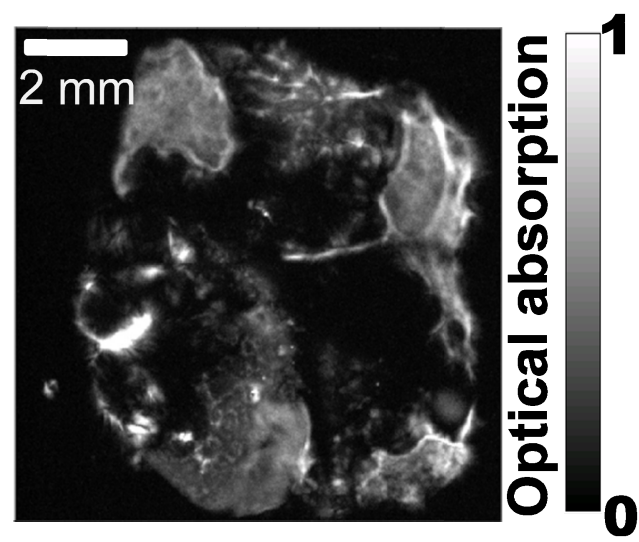

(D)

Fig. 5. Photographs of a healthy tooth $(A)$ and a decayed tooth (B). Dark-field AR-PAM MAP images of the healthy tooth $(C)$ and the decayed tooth (D) showed significant differences in optical absorption contrast.

In order to investigate whether dental pulp tissue can be seen by PAM, an in vitro tooth sample was cut lengthwise to a thickness of $4 \mathrm{~mm}$ in side view (Fig. 6A). A black mark on the top of the tooth sample was used to identify its top surface. This sample was imaged with the NIR PAM system before and after another black mark was painted on the cut surface of this tooth sample (Fig. 6B). Fig. 6C is a MAP image (surface signal removed) from the front before the black mark was painted. Fig. $6 \mathrm{D}$ is a frontal MAP image (surface signal removed) after the 
black mark was painted. The $4 \mathrm{~mm}$ deep, black mark can clearly be seen through the hard dental tissue. Black ink that infiltrated into the crack of the dental tissue is also identifiable in Fig. 6D.

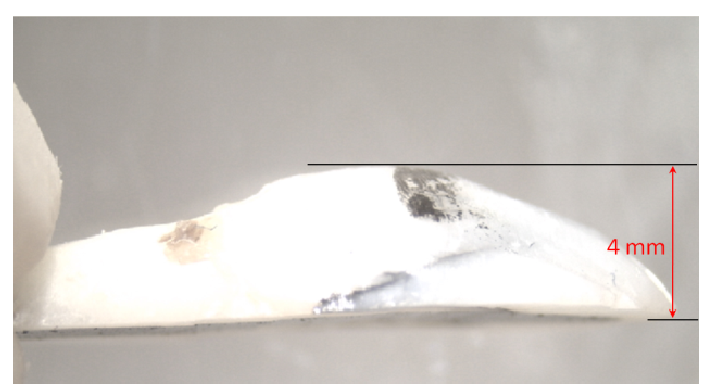

(A)

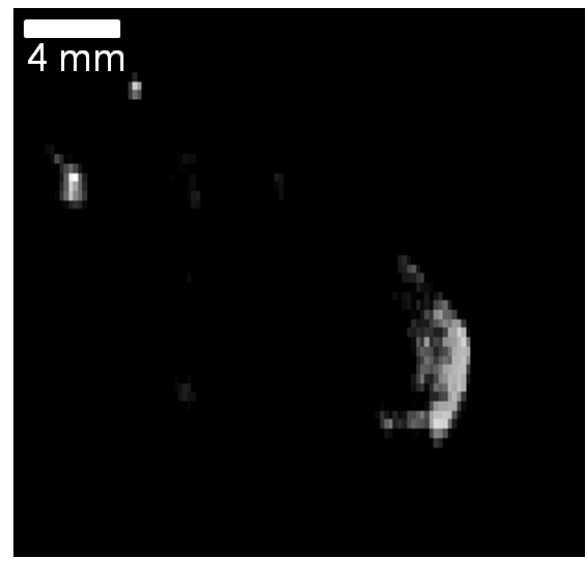

(C)

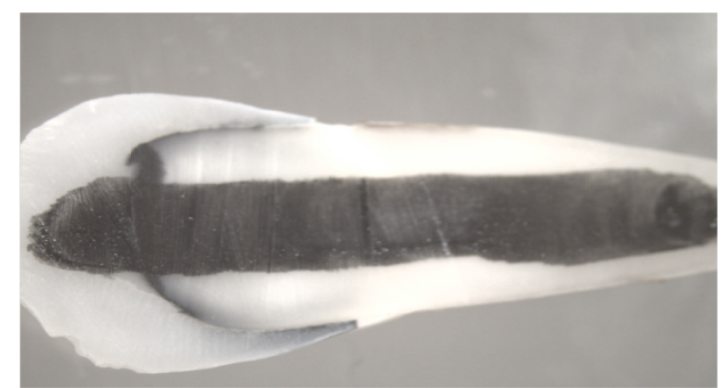

(B)

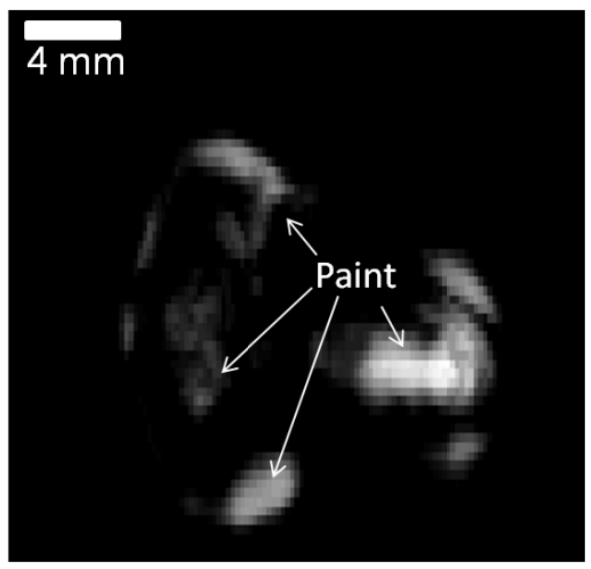

(D)

Fig. 6 An in vitro tooth sample was cut lengthwise to a thickness of $4 \mathrm{~mm}$ in side view (A). A black mark on the top of the tooth sample was used to identify its top surface. For comparison, the tooth was imaged before and after a long, black mark was painted on the cut surface of the tooth sample (B). (C) is a MAP image (surface signal removed) from the front before another black mark was painted on the cut surface. (D) is a frontal MAP image (surface signal removed) after another black mark was painted.

Another in vitro tooth sample was prepared with a 1-mm hole drilled in the tooth center. Before bovine blood was injected into the tooth hole, the tooth sample was imaged as a control. After bovine blood was injected, the tooth sample was imaged again and compared with the control image. The injected bovine blood can be seen in a PAM MAP image that was presented in a poster on Jan $23^{\text {rd }}, 2011$, at the BIOS 2011 meeting. 


\section{Conclusions}

PAM imaging is sensitive to the color change associated with dental decay. Although the relative PA signal distribution may be affected by surface contours and subsurface reflections from deeper dental tissue, monitoring changes in the PA signals (at the same site) over time is necessary to identify the progress of dental decay. It was also demonstrated that deep-imaging NIR PAM can sensitively image blood in the dental pulp of an in vitro tooth. In conclusion, PAM is a promising tool for imaging both dental decay and dental pulp.

\section{References}

[1] L. V. Wang, "Prospects of photoacoustic tomography," Medical Physics 35 (12), 5758-5767 (2008).

[2] Lihong Wang, "Multiscale photoacoustic microscopy and computed tomography," Nature Photon. 3, 503-509(2009).

[3] K. Maslov, G. Stoica, and L. V. Wang, "In vivo dark-field reflection-mode photoacoustic microscopy," Optics Letters 30 (6), 625-627 (2005).

[4] K. Maslov, H. F. Zhang, S. Hu, and L. V. Wang, "Optical-resolution photoacoustic microscopy for in vivo imaging of single capillaries," Optics Letters 33, 929-931 (2008).

[5] K. Song, and L. V. Wang, "Deep reflection-mode photoacoustic imaging of biological tissue," Journal of Biomedical Optics 12, 060503-(1-3) (2007). 